\title{
MAIS OU EST DONC LUTEVA? OU GEOPOLITIQUE D'UNE CAPITALE IMPROBABLE
}

\author{
Pierre Garmy*, Thérèse Panouillères* et Laurent Schneider** \\ avec une contribution de Guilhem Fabre*
}

\begin{abstract}
"La ville de Lodève est située au milieu d'un cirque de montagnes, derniers échelons des plateaux de l'Escandorgue et du Larzac, qui forment un étranglement marqué au confluent de la rivière de Lergue, tributaire de l'Hérault, et du ruisseau important de Soulondres. Il n'est pas douteux qu'une telle situation n'ait, de très bonne heure, déterminé l'occupation de ce lieu à la fois accessible et défendu. Commandant la route du Rouergue au Languedoc, il peut la faire servir à son commerce entre la montagne et la plaine, comme l'intercepter à une ville rivale; et dès lors, centre naturel de la région que dessine la ceinture du bassin de Lergue, il est appelé à la dominer, tandis que, fermé à tout voisinage immédiat, il est aussi bien placé pour conserver jalousement son autonomie. L'histoire de ce pays répondra à ses destinées naturelles ».
\end{abstract}

Ernest Martin

"Histoire de la ville de Lodève »

Montpellier, 1900, p. 1-2.

* UMR 5140, Montpellier-Lattes.

** UMR 6572, Aix-en-Provence.

Au livre III, chapitre IV de son Histoire naturelle, Pline l'Ancien (NH III, 4, 37) dresse la liste alphabétique des oppida Latina de la Provincia Narbonensis. Ainsi figurent, à leur bonne place, des «Lutevani, qui et Foroneronienses », et il fait peu de doute que l'on doive traduire «les habitants de Luteva, qui sont aussi appelés Foronéroniens (ou habitants de Forum Neronis) ». De la sorte l'existence de la ville de ces Lutevani se trouve effectivement attestée, mais elle l'est aussi par l'inscription lapidaire (CIL XII, 4247) découverte près de Béziers et mentionnant la c(olonia ?) Claudia Luteva (Gascou 1995). De même, le sera-t-elle à nouveau, à plusieurs reprises, dans des documents antiques plus récents, «civitas Lutevensium », « id est Luteva castrum » (Not. Gall.), «civittas Lutevensis» (Conc. Gall. : 213, 23), Loteva (Table de Peutinger) (Février 1989 : 62). Comme par ailleurs, le toponyme «Lodève» (Hamlin 1983 : 213) échappe à toute autre origine étymologique que «Luteva» et ses variantes, il faut bien se résoudre, s'inscrivant d'ailleurs en cela dans la tradition historiographique largement dominante (Vinas 1867), à assimiler le site de la ville moderne avec celui de la capitale de Cité antique.

Or, parallèlement, les vestiges archéologiques attribuables sur le terrain à l'établissement urbain antique sont tellement peu nombreux et si faiblement caractéristiques que des localisations alternatives ont jadis été proposées (Creuzé de Lesser 1824 : 222-223). Plus récemment, une partie des recherches entreprises dans le cadre du PCR (projet collectif de recherche) soutenu par le ministère de la Culture «Lodève et son territoire du II ${ }^{\text {èe }}$ âge du fer à la fin du Moyen Âge » a porté précisément sur cette question récurrente, au moyen d'une série d'opérations spécifiques. Les résultats de ces dernières recherches que l'on mettra en perspective avec les données plus anciennes fournissent ici l'occasion d'ouvrir une nouvelle fois le dossier.

1. Bilan des découvertes de vestiges antiques sur l'assiette de la ville médiévale intra-muros

1.1 Le site d'implantation (Contribution G. Fabre)

Le site de Lodève (fig. 1), dont l'axe majeur est d'orientation ouest-nord-ouest - est-sud-est, occupe un espace restreint, en pointe de confluence de deux cours d'eau pérennes du bassin-versant de l'Hérault, nés sur la bordure méridionale du causse du Larzac, la Lergue, le principal, et la Soulondres. Sa genèse s'inscrit essentiellement sous un double contrôle, lithostructural et hydrogéomorphologique. En effet, dans ces hauts bassins-versants, les pentes dont celles des talwegs sont fortes mais se brisent nettement dès qu'elles pénètrent dans le bassin de Lodève, lequel est vigoureusement imprimé dans le canevas structural régional. Ces gradients ont engendré une hydrodynamique à la fois nuancée et efficace en fonction des évolutions paléoclimatiques. Les résultantes sont particulièrement marquées dans la topomorphographie des lieux, où le binôme dépôt-érosion joue à plein. D'une part, on observe une accumulation détritique alluviale relativement importante avec paléotracés d'écoulements épigés plus ou moins bien conservés, dans les zones favorables: pièges sédimentaires, comme les lobes convexes de méandres, les environs des verrous structuraux, les méplats, et les 
creux. Elle est d'âge récent, en gros Pléistocène supérieur — fin du Pléistocène moyen, risso-würmienne en particulier. On a, d'autre part, une incision postérieure verticale dont l'amplitude moyenne est voisine de 10 mètres, par les cours d'eau qui continuent à alluvionner dans leurs lits, d'où leur nette distinction dans le paysage.

La ville se situe sur ces accumulations pléistocènes étagées relativement planes ou à faibles pentes, en position dominante par rapport aux lits actuels des cours d'eau bordiers qui les ont engendrés dans un passé récent. C'est un site on ne peut plus classique d'espace urbain « hors eau », intégré dans un contexte hydrique marqué. La topographie du site est ainsi suffisamment prégnante pour que le recouvrement exact de la ville antique par la ville médiévale puis moderne ait été longtemps proposé sans vérification plus poussée.

Deux synthèses récentes (Schneider, Garcia 1998; Christol et al. 2002) ont fait le point sur les découvertes archéologiques anciennes; on n'en reprendra donc ici que ce qui intéresse directement les problèmes de topographie urbaine, ne retenant que les éléments dont l'identification et la datation ne font pas difficulté (fig. 2).

\subsection{Le quartier à l'est de la rue Neuve des Marchés (anciennement rue du Marché)}

Aucune découverte n'a jamais été signalée dans la partie orientale de la ville enclose, entre le rempart et l'ancien quartier médiéval juif, immédiatement à l'ouest de l'actuelle place du Marché. Même le transfert et la reconstruction de l'église Saint-Pierre en 1851, depuis son lieu d'implantation primitif, place du Marché jusqu'à son emplacement actuel, rue de Lergue, n'ont donné lieu à aucun signalement de vestiges. Il est donc sans doute raisonnable de considérer ce vide comme significatif.

\subsection{Le quartier de la place du marché (fig. 3)}

En 1982, la direction des Antiquités historiques sous la responsabilité de Pierre-Yves Genty, a réalisé une série de sondages aux abords de l'impasse Molinier ; l'un, de quelques $\mathrm{m}^{2}$, a livré au contact avec le substrat un niveau peu caractérisable contenant quelques rares tessons de poterie antique dont deux fragments de céramique sigillée sud-gauloise et un fragment de céramique campanienne. Après la démolition du quartier en 1992, une campagne de diagnostics conduite en 1997 par l'AFAN sous la direction de Françoise Paone, à l'initiative du service régional de l'Archéologie et de la mairie de Lodève, a permis de compléter notablement ces données initiales en confirmant le caractère diffus de l'occupation antique du secteur.

L'opération, couvrant pour la première fois à Lodève plusieurs centaines de $\mathrm{m}^{2}$ d'observations stratigraphiques, n'a pas livré, pour l'Antiquité, de structures bâties en place hormis, en zone 2, un puits (PT 2114), éventuellement attribuable au Haut-Empire, mais qui n'a pas été entièrement fouillé et dont le calage chrono-stratigraphique n'est pas assuré (la relation stratigraphique avec le remplissage 2094 de la fosse 2133 dans laquelle il semble creusé et qui ne contient que du mobilier antique - fond d'amphore Dr 1, anse d'olpé et fond de cruche en pâte claire — ne donne, au mieux qu'un terminus post quem), et, " sous toute réserve ", une maçonnerie (2007), large d'un mètre environ, orientée grosso modo à $45^{\circ}$ ouest NL et interprétée par les fouilleurs comme un mur de terrasse (Paone 1997 : 46). Il convient également de mentionner la présence en zone 1 d'une fosse quadrangulaire (1085), creusée dans le substrat, partiellement fouillée, qui a livré un mobilier homogène datable du Haut-Empire (sigillées sud-gauloises, parois fines, céramiques à pâte claire, et 1 tesson de céramique campanienne A au sommet du comblement).

Mis à part ces trois vestiges peu sûrs, l'occupation antique des lieux est représentée, au nord du site (zone 1) par des niveaux non structurés, limono-argileux et sableux et, au sud par un mince niveau général de terre rougeâtre contenant du cailloutis au contact de la terrasse superficiellement remaniée (US 2006, 2075 et 2130 de la zone 2, US 3182, 3206 et 3218 de la zone 3) qui contiennent tous du mobilier céramique du HautEmpire, homogène mais extrêmement fragmenté.

La nature et la densité des vestiges antiques découverts sur la place du Marché permettent de justifier sans conteste l'opinion des fouilleurs (Paone 1998 : 25) pour qui « il semble possible de dire que la ville romaine de Luteva ne s'étendait pas dans cette partie » du tissu urbain moderne, « les traces d'occupation, sommaires et ponctuelles, [...] témoignant d'une implantation lâche dans une zone non urbanisée » (Paone $1997: 25$ ).

\subsection{Square Georges Auric (ancien parvis de l'hôtel du Cardinal de Fleury)}

En prévision d'une restructuration urbaine aux abords de l'ancien hôtel du Cardinal de Fleury, Pierre-Yves Genty en 1982, puis Laurent Schneider en 1985 ont effectué une série de sondages stratigraphiques dont les résultats sont complémentaires. On en trouvera une relation dans Catalogue (1998: 19-21) et, de manière plus détaillée, dans Schneider, Garcia (1998: 211-213). D'une superficie limitée à quelque $70 \mathrm{~m}^{2}$ en tout, la double intervention des années 1980 a livré, en centre ville, une série d'horizons stratigraphiques bien datés entre la première moitié du $\mathrm{I}^{\mathrm{er}}$ s. et le III ${ }^{\mathrm{ème}}$ s. ap. J.-C., associés à divers vestiges bâtis (fig. 4a et b). 
$\mathrm{Au} \mathrm{I} \mathrm{e}^{\mathrm{er}}$ siècle, apparemment dès la période augustéenne, appartiennent les structures suivantes :

- Un niveau argileux (US 2006 du sondage 2000, 1985) au contact du substrat, dans lequel est creusée une fosse aménagée pour implanter un dolium.

- Une fosse rectangulaire creusée dans le substrat et tapissée d'argile (C9b du sondage 7, 1982), associée à un fossé rectiligne - dont le prolongement a été observé dans le sondage 6 , à quelques mètres plus au sud. Associés à cette structure excavée, des niveaux de colmatage des irrégularités du substrat $(\mathrm{C} 9, \mathrm{C} 8$, C4f du sondage 7) et la semelle de fondation d'un mur lié au mortier. Dans un second temps (seconde moitié du I ${ }^{\text {er }}$ s. ap. J.-C.), fosse et fossé sont comblés et-ou arasés et font place à une surface empierrée interprétée par les fouilleurs comme un espace de circulation — rue ou place, l'exiguïté des sondages n'a pas permis de trancher.

- Une structure empierrée avec liant de mortier, très mal conservée et donc non identifiable (US 1014 du sondage 1000, 1985), surmontée d'un niveau de circulation fait de l'épandage d'un dépotoir domestique (US 1010).

Sans solution de continuité semble-t-il, le secteur est réaménagé dans le courant du II $^{\text {ème }}$ siècle, voire au début du III ${ }^{\text {ème }}$.

Le sondage 4 de 1982 et les sondages 2000 et 3000 de 1985, implantés volontairement dans la continuité du premier, ont révélé la présence d'un bâtiment dont on a reconnu quelques éléments : de part et d'autre d'un mur, fondé jusqu'au substrat et qui a donc recoupé certaines des structures décrites ci-dessus (US 2006), un sol de terre battue à l'est et un sol de tuileau à l'ouest (tous deux très mal conservés) marquent la présence de deux pièces dont il est difficile de préciser la fonction. Les deux sols sont construits sur un niveau de réglage constitué sans doute, pour part de remblais et, pour part de l'aplanissement des installations plus anciennes. C'est ce qui peut expliquer le large éventail chronologique du mobilier rencontré dans cet horizon qui couvre largement les deux premiers siècles de notre ère. Un as de Septime Sévère découvert en 1985 fournit un terminus post quem à l'aménagement du bâtiment. La nature des découvertes mobilières indique plutôt un contexte domestique sans que l'on puisse aller plus loin dans l'interprétation. Seul élément discordant, un pied de siège en marbre blanc en forme de patte de griffon découvert en remblai dans la couche de réglage du sol ouest (US 2002-2005) et qui évoque un aménagement balnéaire ou un édifice de spectacle dont cependant aucun autre élément n'a été découvert.

Certains secteurs du quartier peuvent avoir été mis en culture précocement comme en témoignent les US 1009, 1007 et 1006 (fin du $\mathrm{I}^{\text {er }}$ et II ${ }^{\text {ème }}$ siècle). Toujours est-il qu'aucun témoin mobilier postérieur au III ${ }^{\text {ème }}$ siècle ne vient indiquer une continuité d'occupation du quartier durant l'Antiquité tardive et le haut Moyen Âge. Il faut toutefois noter que les fouilleurs, en 1982 comme en 1985, ont remarqué la présence généralisée, audessus des niveaux de destruction des structures du Haut-Empire, d'« une puissante couche sombre très organique, d'une épaisseur de $1 \mathrm{~m}$, (qui) indique que les lieux ont été voués pour l'essentiel à des espaces de jardins jusqu'à la fin de l'époque moderne ». La description suggère une parenté assez proche avec les «terres noires » que l'on commence à mieux connaître dans les villes du nord de l'Europe (Cammas et al. 1995 ; Galinié dir. 2000 ; Galinié 2002).

Rue de la République, au moment de son percement en 1891, dans la section comprise entre la Grand rue et la rue Vieille Commune, soit à quelques dizaines de mètres seulement au nord nord-ouest des sondages devant l'hôtel de Fleury, Ernest Martin (1900:316) rapporte la trouvaille de « quelques vases en terre blanche d'environ 1,50 $\mathrm{m}$ de haut, très pointus à la base [...] (qui) paraissent être des amphores... ». La description est malheureusement trop lapidaire pour préciser de quel type d'amphore il peut s'agir et a fortiori définir leur fonction à l'endroit de leur découverte même si on peut en déduire qu'elles devaient être alors à peu près entières.

\subsection{Cathédrale et quartier de l'Hôtel de ville}

En 1980, l'installation du chauffage par le sol dans la cathédrale a entraîné la mise au jour « fortuite » de sépultures et de constructions. La direction des Antiquités historiques est alors intervenue sous la conduite de P.-Y. Genty pour mener, dans des conditions très difficiles une fouille de sauvetage sur une partie du chevet de l'église et sur le bras septentrional du cloître, transformé en chapelles (Catalogue 1998 : 15-18; Schneider, Garcia 1998 : 209-211). L'opération a mis au jour une partie d'une crypte ancienne liée à un état de l'édifice cathédrale antérieur au bâtiment actuel, et plusieurs ensembles funéraires datés entre le haut Moyen Âge et le $\mathrm{XVIII}^{\text {̀̀me }}$ siècle.

Le niveau sépulcral le plus ancien, fouillé dans l'aile du cloître, comportait sur la faible emprise fouillée, un sarcophage monolithe trapézoïdal à logette céphalique et couvercle en bâtière basse, décoré de six boutons sommitaux (tombe 2) et deux autres sépultures (tombes 8 et 9) en dalles de schiste posées de champ, dont l'une équipée également d'une logette céphalique. En l'absence de mobilier datable, seule la typologie régionale fournit une indication chronologique pour cet ensemble dans un large haut Moyen Âge. Il faut sans doute rattacher au même contexte trois chapiteaux cubiques découverts en remploi (Nougaret 1975 : 108-109; 
Schneider, Garcia 1998 : fig. 153-155) le tout contribuant, modestement, à esquisser les traits d'un ensemble cathédrale des $\mathrm{V}^{\text {ème }}-\mathrm{VII}{ }^{\text {ème }}$ siècles.

Les inhumations anciennes sont pratiquées à travers des niveaux antiques homogènes datés entre l'époque augustéenne et le IV ${ }^{\text {ème }}$ siècle. Au contact du substrat, le premier niveau contient exclusivement du mobilier céramique augustéen, roulé, très fragmentaire et sans relation avec un sol en place, ce qui a fait penser aux fouilleurs qu'il devait s'agir d'un « épandage à vocation agricole dans des terres cultivées très proches de la ville ». En revanche, le niveau immédiatement supérieur était plus clairement structuré et en place avec un mobilier datable entre l'extrême fin du II ${ }^{\text {ème }}$ s. et le IV ${ }^{\text {ème }}$ s. ap. J.-C. La superficie infime du sondage n'a pas permis de préciser la nature de l'occupation correspondante.

Il n'est sans doute pas indifférent de remarquer que les séquences chronologiques antiques rencontrées dans les sondages sous la cathédrale Saint-Fulcran, sont conformes à celles observées aux abords de l'hôtel de Fleury.

En 1982, P.-Y. Genty a pratiqué par ailleurs un sondage stratigraphique contre le parement intérieur de l'enceinte médiévale, immédiatement au nord de l'ancien palais épiscopal, aujourd'hui siège de la mairie de Lodève. Aucune structure antique en place n'a été relevée, mais tous les niveaux médiévaux liés à la construction du rempart ont livré un abondant mobilier du Haut-Empire.

Enfin, E. Martin (1900 : 316) signale la découverte à la fin du XIX ${ }^{\text {ème }}$ siècle, place Broussonnelle (aujourd'hui place Alsace-Lorraine, anciennement Bossanelle) à deux mètres de profondeur, d'ossements «mêlés avec des briques cassées et autres débris » ainsi qu'un stylet «que nous avons reconnus tout de suite pour être de l'époque romaine ». On peut sans doute accréditer sa proposition de datation du mobilier mais pas celle des ossements, à propos desquels l'auteur a d'ailleurs « soutenu qu'on n'avait aucune certitude que ce fût des ossements de chrétiens ». En effet, la mise au jour, en 1892, toujours sous la place de «cercueils en ardoise garnis d'ossements » (Martin $1900: 316$ ) suggère davantage, malgré le manque de précision de la description des sépultures, la présence d'un cimetière médiéval éventuellement en relation avec le couvent de Nonenque et l'église Saint-André mitoyens (Demaille 2000 : 178) ou bien des sépultures du même type que celles décrites cidessus sous le cloître et donc à dater du haut Moyen Âge.

2. Hypothèse d'une implantation urbaine sur les basses pentes du Grézac (Prospections 1999-2000 et sondages mécaniques 2002)

L'assiette de l'agglomération antique se trouve ainsi singulièrement réduite par rapport à l'idée de départ d'une cö̈ncidence avec celle de la ville close du Moyen Âge. Il convenait donc de vérifier sur le terrain une hypothèse alternative selon laquelle la ville antique pourrait se situer plus au nord-ouest et prendre place sur les reliefs qui dominent le site du confluent Lergue-Soulondres, depuis le plateau du Grézac jusqu'à la caserne de gendarmerie qui le borde à l'est. Une campagne de prospections de surface a donc été entreprise durant l'hiver 1999-2000 dans ce secteur. Elles ont été menées suivant deux paliers d'altitude, entre 300 et $400 \mathrm{~m}$ environ autour du lieu-dit Grézac d'une part, entre 250 et $150 \mathrm{~m}$, plus proche du centre ville aux lieux-dits Font Bonne et Versailles d'autre part. Le mitage récent de ces collines par un habitat individuel diffus rend ce type d'opération difficile, le nombre de terrains libres pour la prospection étant extrêmement réduit. C'est pourquoi, on a été obligé de se contenter d'un échantillonnage minimal de la zone où toute prospection systématique est désormais exclue.

Cinq secteurs (fig. 5) ont été prospectés en détail : deux au lieu-dit Grézac (1 et 2), deux à Versailles (3 et 4) et un à Font-Bonne (5). Les quatre premiers se sont révélés entièrement stériles en documents anciens. À Font Bonne, on a collecté le mobilier datable suivant (détermination C. Sanchez) :

— Sigillée du sud de la Gaule (SIG-SG) : 5 fragments (pour un poids total de $14 \mathrm{~g}$ ) dont 1 fragment de Dr15/17 .

- Verre : 2 fragments sans forme

On a donc affaire à un lot restreint mais chronologiquement homogène de la première moitié du $\mathrm{I}^{\mathrm{er}} \mathrm{s}$. de notre ère. Toutefois, le nombre très limité de tessons et le manque de diversité des mobiliers rencontrés interdisent probablement de voir à cet endroit une occupation in situ de type urbain. On notera en particulier l'absence de matériaux de construction et de mobiliers antiques autre que la vaisselle fine. Le fait que les deux autres prospections réalisées à peu de distance à l'est (lieu-dit Versailles) n'aient livré aucun vestige milite dans le même sens. Au mieux, peut-on voir dans ce très modeste lot, s'il ne s'agit pas purement et simplement des restes d'un amendement agricole, l'image d'un petit site isolé, en rapport avec la voie qui le borde au sud (fig. 5) et qui correspond à l'un des «camis ferrats » reliant Lodève au causse par la crête de l'Escandorgue. C'est d'ailleurs dans ce secteur, au lieu-dit Saint-Martin, soit à moins de $200 \mathrm{~m}$ plus au sud, que se trouve le cosi detto mausolée de l'Antiquité tardive (infra 5).

En outre, trois sondages mécaniques pratiqués (fig. 5 et 6) lors de la campagne organisée au printemps 2002 (infra 3.3 et 3.5) viennent compléter ces observations de surface (tranchée 20 à l'ouest du stade, P. C. 366, 
section AD - tranchées 16 et 17, lieu-dit Versailles, P. C. 538, section AD). Tous se sont révélés stériles en documents anciens.

Dans le même programme, une prospection de contrôle a été menée au sommet de la colline de Montbrun, en rive droite de la Lergue et de la Soulondres, sur les seuls terrains accessibles situés immédiatement à l'ouest du château médiéval (fig. $5:$ 6). Celui-ci a fait l'objet dans les années 1980 de plusieurs campagnes de fouilles qui ont mis en évidence les bases d'une tour médiévale et plusieurs autres murs, dont un mur en moyen appareil de grès que la tradition érudite locale (Catalogue 1998 :23-24) persiste à dater de l'antiquité alors même qu'aucun sondage exécuté sur le plateau n'a révélé de niveaux antiques en place (Gallia Informations 1987-88 $1: 251)$.

Les nouvelles prospections ont livré le mobilier suivant (détermination C. Sanchez) :

- $\quad$ Sigillée du sud de la Gaule (SIG-SG) : 9 fragments (pour un poids total de 18 g.) dont 1 fragment de Dr15/17, 1 fragment de Dr27, 1 fragment de Dr29 (?), 1 fragment de Dr37, 1 fragment de He 7 (?), 4 fragments de forme indéterminée dont deux portant un décor.

- Céramique commune sableuse (SABL-OR) : 1 bord proche de la forme B-O-B A1.

Ce cortège indique plutôt un horizon de la fin du $\mathrm{I}^{\mathrm{er}}$ s. ou du II ${ }^{\mathrm{ème}}$ s. ap. J.-C. mais la fourchette chronologique complète est plus large. Vu le nombre infime de tessons, leur indice de fragmentation très élevé, leur datation assez lâche, on aura tendance à interpréter ces vestiges comme ceux d'apports pour amendements et non comme les témoins d'une occupation sur place. Ces résultats confirment les conclusions antérieures qui font du site de Montbrun un établissement qui ne remonte pas au-delà du castellas médiéval et qui reste, de toute façon, extérieur à l'assiette urbaine du site d'interfluve, en rive gauche de la Soulondres.

3. Hypothèse d'une extension urbaine antique hors les murs de l'enceinte médiévale, jardin public, parc municipal, esplanade (Campagne de sondages mécaniques 2002)

$\mathrm{Au} \mathrm{vu} \mathrm{du} \mathrm{résultat} \mathrm{des} \mathrm{recherches} \mathrm{sur} \mathrm{le} \mathrm{versant} \mathrm{oriental} \mathrm{du} \mathrm{Grézac,} \mathrm{il} \mathrm{restait} \mathrm{à} \mathrm{vérifier} \mathrm{une} \mathrm{ultime}$ conjecture, celle de l'éventuelle extension de la ville antique à l'extérieur de l'enceinte médiévale, au nord de la cathédrale et de l'ancien palais épiscopal, sur une vaste zone à peu près inexplorée sur le plan archéologique qui s'étale depuis l'Esplanade et le jardin public ou parc municipal - correspondant aux anciens jardins de l'évêché —, en direction de la gendarmerie et ses abords, depuis la route de Grézac jusqu'au quartier de Versailles (fig. 6). Cette extension présumée, qui couvre en tout, avec l'emprise de la ville médiévale, une superficie approximative de 22 ha, était, récemment encore, la plus communément admise : on la trouve notamment cartographiée dans Christol et al. (2002 : 286) et elle a aussi servi de base dans une étude sous presse concernant les structures du peuplement antique du territoire de la Cité (Garmy et al. sous presse).

Pour tester cette dernière hypothèse de travail, il s'est avéré possible au printemps 2002, grâce à la bonne volonté de la mairie de Lodève et l'aide efficace de ses services techniques, de réaliser, hormis les trois tranchées déjà mentionnées, une série de 18 sondages mécaniques, non destructifs, destinés à vérifier la présence ou l'absence de niveaux gallo-romains en place dans ce secteur de la ville. Pour obtenir un échantillon topographiquement représentatif, les sondages ont été distribués largement sur l'ensemble du Parc municipal (P. C. 224, section $\mathrm{AD}, 13$ tranchées) et alentour sur tous les terrains municipaux accessibles sans difficulté avec un engin mécanique : lieu-dit Versailles (P. C. 538, section AD, 2 tranchées), avenue de Prémerlet (P. C. 636, section $\mathrm{AD}, 3$ tranchées) et Esplanade (P. C. 431 et 432, section AD, 2 tranchées). Les tranchées-sondages ont été effectuées à l'aide d'un tractopelle en mode rétro, équipé d'un godet plat de 1,20 m de large. Toutes ont été menées jusqu'au substrat géologique en l'absence de structure en place, ou jusqu'à l'affleurement de celles-ci lorsqu'il y en a.

\subsection{Parc municipal (fig. 6)}

Une première série de 6 tranchées numérotées de 1 à 6 a pris place selon deux axes au sud du jardin public.

- Tranchée 1

Environ 7 mètres de long. Ouverte jusqu'au substrat : aucune structure en place.

- Tranchée 2

Environ 10 mètres de long. Ouverte jusqu'au substrat : aucune structure en place.

- Tranchée 3

Environ 13 mètres de long. Présence d'une surface empierrée de circulation d'époque moderne (chemin ?), à 70 $\mathrm{cm}$ de profondeur sous le sol actuel.

- Tranchée 4

Environ 6 mètres de long. Ouverte jusqu'au substrat : aucune structure en place.

- Tranchée 5 
Environ 10 mètres de long. Présence, à $50 \mathrm{~cm}$ de profondeur, d'une structure bâtie non identifiée et non datée faite de quelques blocs calcaires grossièrement équarris et de lauzes de schiste. Les niveaux de sédiments en liaison avec la structure contiennent un rare mobilier moderne et contemporain.

- Tranchée 6

Environ 6 mètres de long. Ouverte jusqu'au substrat : aucune structure en place.

Une tranchée isolée (12) a été ouverte au centre du parc, à peu de distance du bassin.

- Tranchée 12

Environ 3 mètres de long. Ouverte jusqu'au substrat : aucune structure en place.

Une deuxième série de 6 sondages (tranchées 7 à 11 et 21) a été effectuée dans l'angle nord-est du jardin, à proximité des Allées de la Résistance, entre le monument aux Morts de Dardé et le boulodrome.

- $\quad$ Tranchée 7 (fig. 7)

Environ 14 mètres de long. On a relevé la stratigraphie suivante, de haut en bas :

○ 1701, 1702,1703: Horizons de terre végétale mêlée à des matériaux hétérogènes (pierres et gravillons).

- 1704 : Limon sableux, brun clair avec gravillons et traces de mortier (hétérogène).

- 1705 : Limon brun moyen, homogène avec présence de blocs.

○ 1706 : Limon brun moyen associé à de nombreux éléments de mortier blanc pilé et de petits éclats de grès.

- 1707 : Repérée uniquement en sondage profond à l'extrémité nord de la tranchée. Limon sombre, homogène et anthropisé (traces de charbons de bois).

- 1708 : Limon argileux compact avec mobilier antique (éclats de tuile) et traces de charbons de bois.

- 1709 - Au nord-est du mur 1715, sous 1706. Cailloutis, pierres, blocs et tuiles dans un sédiment argileux brun clair compact $(=1713)$.

○ 1710 - Sédiment argileux homogène contre le mur 1711 au nord-est $(=1712)$.

- 1711 - Mur à l'extrémité sud-ouest de la tranchée. Au dessus d'une assise grossièrement appareillée faite de blocs calcaires tout-venant, assise traversante complète de 2 plaques de schiste soigneusement taillées, de $3 \mathrm{~cm}$ d'épaisseur en moyenne.

- 1712 - Contre le mur 1711 au sud-ouest (=1710). Présence de céramique dont 1 frag. de bord de SIG-SG Dr17a brûlé.

- 1713 - Horizon brun clair, argileux et compact recouvrant un éboulis non fouillé. Présence de céramiques (tessons de SIG-SG non identifiables, 1 frag. de CLAIR-B 68 et 1 frag. d'AF-CUI 23B +2 frag. de verre + bronze).

- 1714 - Mur? Alignement de blocs de pierres avec présence de 2 frag. de SIG-SG (1 bord et 1 fond d'assiette datables 60-150), de fragments de tuile et de petits blocs de mortier.

○ 1715 - Mur.

- 1716 - Lentille cendreuse en interface entre 1706 et 1710/1713. Observée uniquement en coupe.

- $\quad$ Tranchée 8 (fig. 8)

Environ 9 mètres de long. De même orientation que la tranchée 7 mais distante de quelques 15 mètres.

○ 1810 - Limon compact avec mortier. (Fouillé). Présence de céramique dont essentiellement quelques tessons de SIG-SG avec 1 frag. SIG-SG Dr37a et 1 frag. SIG-SG VeA1, plus 1 frag. de bord de couvercle SABL-OR E3 et un frag. de bord en bourrelet de SABL-OR A3.

- 1811 - Tranchée de récupération (?) creusée dans 1810. Datation indéterminée.

○ 1812 - Sondage sous une dalle dans le sédiment brun compact. Datation indéterminée.

- Tranchée 9

Environ 5 à 6 mètres de long. Orientée est-ouest le long du monument aux Morts. Ouverte jusqu'au substrat : aucune structure en place.

- $\quad$ Tranchée 10 (fig. 9)

Environ 6 à 7 mètres de long. Présence d'un mur.

- Tranchée 11

De 4 à 5 mètres de long. Ouverte jusqu'au substrat : aucune structure en place.

- $\quad$ Tranchée 21 (fig. 9)

Environ 6 mètres de long. Présence d'un mur (?) dans l'alignement de celui observé dans la tranchée 10.

3.2 Avenue de Prémerlet (Section AD, P. C. 636) (fig. 6)

Trois tranchées ont été ouvertes. Deux, dans l'angle nord-est de la parcelle (tr. 13 et 14), la dernière, au sud-est (tr. 15). 
- Tranchée 13

Près de 11 mètres de long. Ouverte jusqu'au substrat : aucune structure en place.

- $\quad$ Tranchée 14

Environ 15 mètres de long. Ouverte jusqu'au substrat : aucune structure en place.

- Tranchée 15

De 4 à 5 mètres de long. Ouverte jusqu'au substrat : aucune structure en place.

3.3 Lieu-dit Versailles (Section AD, P. C. 538) (fig. 6)

Deux tranchées ont été ouvertes en bordure de la parcelle, le long du ruisseau du Petout (tr. 16 et 17).

- Tranchée 16

Environ 5 mètres de long. Substrat non atteint : aucune structure en place.

- $\quad$ Tranchée 17

Environ 5 mètres de long. Substrat non atteint : aucune structure en place.

3.4 Esplanade (Section AD, P. C. 431 et 432) (fig. 6)

Deux tranchées ont été ouvertes, dans le jardin du Trésor public, dans l'angle est de la parcelle, le long de la rue de la Sous-Préfecture (tr. 18 et 19).

- $\quad$ Tranchée 18

Plus de 5 mètres de long. Ouverte jusqu'au substrat : aucune structure en place.

- $\quad$ Tranchée 19

Environ 6 mètres de long. Ouverte jusqu'au substrat : aucune structure en place.

\subsection{Lieu-dit Versailles (Section AD, P. C. 366) (fig. 6)}

Une longue tranchée y a été ouverte derrière les bâtiments de la caserne des pompiers (tr. 20).

- Tranchée 20

Plus de 7 mètres de long. Ouverte jusqu'au substrat : aucune structure en place.

Les enseignements de la série de sondages effectués en 2002 sont nécessairement limités dans la mesure où il s'agissait de sondages volontairement non destructifs qui n'ont donc pas permis la fouille des niveaux en liaison stratigraphique directe avec les structures construites rencontrées. Au demeurant, celles-ci restent en nombre réduit et sont limitées topographiquement à une zone circonscrite étroitement au plus près de la cathédrale et de l'Hôtel de ville. Tous les sondages entrepris en dehors de cette zone restreinte se sont révélés stériles.

Bien que les structures en question ne soient pas datées stratigraphiquement, il convient cependant de souligner que les horizons sédimentaires qui les jouxtent directement ou même qui les recouvrent, contiennent uniquement un mobilier céramique datable du $\mathrm{I}^{\mathrm{er}}$ au III ${ }^{\mathrm{ème}}$ siècle à l'exclusion de tout témoin plus tardif. Quelques vestiges erratiques plus anciens ont également été rencontrés dans les tranchées 7 et 8 qui se sont révélées les plus riches en documents. Enfin, l'ensemble des architectures mises au jour dans les tranchées 7, 8, 10 et 21 semblent s'organiser selon une orientation unique, qu'il conviendra de préciser ultérieurement, la longueur mise au jour ne permettant pas de les caler sérieusement. La réfection complète du parc municipal, avec notamment le remplacement systématique des espèces de haute tige, permettra sans doute prochainement de pratiquer des fouilles en extension sur la totalité de cet espace.

\section{Nécropoles et découvertes à caractère funéraire (fig. 10)}

Pour la plus grande partie de l'Antiquité, jusqu'à ce que « les morts entrent en ville », on retient comme marque certaine des limites urbaines, l'emplacement des sépultures et des nécropoles, à la périphérie du pomerium, généralement le long des voies. Dans le cas de Lodève, les découvertes d'ordre funéraire ne présentent pas un caractère très démonstratif à ce sujet; il s'agit uniquement d'observations anciennes, en nombre faible, accompagnées d'un signalement souvent très approximatif, y compris sur le lieu précis d'implantation.

Nous présentons ici l'état du dossier en adoptant le sens horaire autour de la ville.

- Aucune découverte n'a jamais été faite sur la rive droite de la Soulondres, au droit de la ville.

- À $1 \mathrm{~km}$ environ à l'ouest de la ville, au bord de la route départementale 35, Guy Barruol a mis au jour en 1963 (supra 2) les restes d'un bâtiment de plan rectangulaire de 14 m x 10 m environ, aux puissantes maçonneries fortement contrefortées, qu'il a prudemment proposé d'interpréter comme un mausolée monumental de l'Antiquité tardive (Gallia 1964-2: 493). Mais l'absence de tout 
indice probant sur la fonction de l'édifice comme sur sa datation le rend lui-même circonspect et il faut sans doute retenir sa réserve.

- Sur la «partie supérieure de l'Esplanade », Martin (1900:317) rapporte d'après Vinas (1867:47), la mise au jour d'un « cippe funéraire » dont la forme « est évidemment romaine » et qui fut déposé alors contre les murs de la cathédrale. (fig. 11 : A). Aujourd'hui conservé au musée Fleury, ce cippe est accompagné d'un autre (fig. 11: B) provenant de Lodève, sans plus de précision sur les inventaires.

- Au quartier de Prémerlet, lieu-dit Blazou, sur le bord d'un ruisseau qui ne peut être dans ce secteur que le Petout, petit affluent intermittent rive droite de la Lergue mais dont le lit est assez profondément incisé dans la terrasse, Martin (1900 : 317), toujours sur les indications de Vinas, décrit la trouvaille de «quantités considérables de briques à rebords et de poteries rouges ». Il estime que cette découverte pourrait être la même que celle décrite pratiquement dans les mêmes termes au «quartier de Grézac » avec abondance de sigillée figurée, une hache et une clef ainsi qu'une petite «mosaïque à double face» (?) dont un côté représente une poule. Aucune mention d'ossements et donc, rien n'indique sans conteste mais n'exclut pas davantage, l'appartenance de ce mobilier à un ensemble funéraire. Cependant les tranchées-sondages 13 et 14 d'une part, 16 et 17 d'autre part, décrites plus haut et situées à proximité immédiate du Petout ont montré l'absence totale de vestiges structurés dans ce secteur.

- Encore plus problématiques sont les mentions des découvertes de la place Neuve, anciennement place aux Herbes, aujourd'hui rue Neuve des Marchés (Martin 1900:313) : «une tombe bien reconnaissable par sa construction » et, à quelques pas de là, quatre blocs parallélépipédiques en grès « joints ensemble sans ciment ni mortier, ce qui indique bien une construction romaine, de telle manière qu'elles (les pierres) formaient entre elles un carré parfait de $1,60 \mathrm{~m}$ de côté sur $0,65 \mathrm{~m}$ de hauteur ». L'extension supposée de la ville antique à l'est, n'interdit pas de porter crédit aux propositions de Martin, mais les précisions qu'il donne sont décidemment insuffisantes pour s'en assurer.

- En revanche, la description des vestiges et du mobilier mis au jour à plusieurs reprises (Martin 1900 : 312-316) dans l'ancien faubourg des Carmes, en rive gauche de la Lergue donc et en bordure de la voie qui longe la rivière (aujourd'hui avenue Denfert et avenue du Général de Gaulle) laisse peu de doute sur la présence d'une ou plusieurs nécropoles antiques à cet endroit.

○ «Vers 1844, en travaillant sur la route de Montpellier [...] à peu de distance du pont de la Lergue, au-dessous du jardin de M. Eugène Brun, des tombeaux, des lacrymatoires en verre, de grosses briques romaines et diverses poteries en terre fine et rouge ».

- Apparemment au même lieu ou très près, devant la maison de famille Brun, « lors de la construction de l'égout de la route de Montpellier, des ossements humains en grande quantité, un long et large cercueil de plomb, à couvercle plat, renfermant un squelette entier» accompagné "d'une coupe de terre rougeâtre très lisse, à bordure de piques allongées » (possiblement SIG-SG Dr24-25 ou SIG-SG VeA ?).

- En 1855, lors de la construction de l'usine Mellet, attenante à l'abattoir public sur la route de Montpellier, lieu bien identifiable aujourd'hui avec le terrain cadastré section AI, P. C. 380, «un fossé ou chemin creux, d'un mètre de section, rempli de cendres et de charbons... » que Martin (1900 : 314-315) interprète comme provenant d'une «maison incendiée ». La suite de sa description ne laisse cependant planer aucune incertitude sur l'identification d'une nécropole, contenant inhumation(s) et incinérations, dont au moins une incinération primaire et sans doute des enclos funéraires. On peut en juger à partir des éléments produits: le fossé, où «se trouvèrent une infinité d'objets de provenance romaine, bien reconnaissables, notamment un squelette d'homme parfaitement conservé, [...] couché sur le côté droit, les genoux touchant presque le menton »; et plus loin : « les objets furent trouvés en abondance, dans le même fossé et mêlés avec les cendres et les charbons, tels que patères en verre irisé, lacrymatoires, amphores en verre en forme de boule [...] une amphorette, deux lampes, quatre pieds d'homme en bronze de $0,10 \mathrm{~m}$ de long, chaussés de cothurne dont le bas de la jambe formant douille encore remplie de bois carbonisé $[\ldots]$ deux lances en fer à un seul tranchant, une fermeture en bronze, une petite coupe en terre rouge, deux anneaux en bronze, le ventre et le pied d'une aiguière en bronze et une infinité de débris en verre et en terre... ». En outre, dans le même jardin, à un mètre de profondeur, " on avait découvert dans le temps plusieurs tombes romaines avec des monnaies de Dioclétien et de Domitien ».

- «Les mêmes trouvailles ont été faites sur l'emplacement de l'usine à gaz, mais les ouvriers, ignorants ou cupides, ont tout brisé et tout dissipé, selon l'usage ordinaire » (Martin 1900 : 316). Le terrain, aujourd'hui encore propriété d'EDF-GDF, cadastré section 
AI, P. C. 24, 25 et 26) se situe à environ $200 \mathrm{~m}$ au sud du précédent. Il peut donc s'agir de la suite de l'ensemble funéraire précédent ou d'un autre du même type, toujours le long de la voie en rive gauche de la Lergue. Tout ce secteur est inscrit dans le périmètre d'une ZAC à réaliser en accompagnement de l'aménagement en cours de l'autoroute A 75 et de l'entrée de ville. Il sera, bien entendu, d'une grande importance d'y conduire les recherches archéologiques appropriées pour mieux caractériser une occupation funéraire à l'évidence étendue et d'un intérêt majeur dans le contexte régional.

$\mathrm{Au}$ total, si la topographie des découvertes funéraires périurbaines est de peu de secours pour cerner précisément le périmètre de la ville antique, elle permet néanmoins, malgré le caractère lacunaire de la documentation, de confirmer que sur ce chapitre Lodève entre parfaitement dans le schéma classique d'une disposition des lieux d'enfouissement le long des principales voies rayonnant autour du noyau urbain.

\section{5. Évaluation du potentiel urbain antique}

En raisonnant par inclusion-exclusion à partir de la teneur et de l'emplacement de toutes les découvertes archéologiques faites à Lodève que l'on vient de recenser, il devient possible de définir à grands traits les contours de l'assiette urbanisée antique (fig. 12).

- À l'ouest, les travaux conduits dans la cathédrale au début des années 1980 et ceux de 2002 dans le jardin public, incitent à fixer une limite qui ne déborde que d'une centaine de mètres au plus celle marquée par l'enceinte médiévale, encore sensible aujourd'hui dans le paysage, même si le mur luimême a disparu.

- À l'opposé, côté oriental, l'absence constante de signalement de découvertes au delà de l'actuelle rue Neuve des Marchés et surtout les résultats des recherches de 1997 permettent d'exclure toute cette partie de la ville médiévale du périmètre plus ancien. Dans ce secteur de la ville, la structure en double patte d'oie, qui se résout à hauteur de l'ancienne place aux Herbes (patte d'oie formée à l'est par les rues de Lergue, des Jacobins et du Mazel, à l'ouest par la rue du Cardinal de Fleury et la Grand Rue), ne peut être mise en relation avec un obstacle naturel, de type chenal ancien de la Lergue, comme on a pu être tenté de la faire naguère (Schneider 2003 : 220). L'étude structurale (supra 1.1) montre en effet clairement que les écoulements sur la terrasse sont tous nettement antérieurs aux périodes qui nous concernent ici. Mais il apparaît également impossible, en raison des résultats des fouilles de $1997 \mathrm{du}$ quartier du marché, de la mettre en relation avec une limite physique de la ville antique du côté oriental. Sans argument décisif mais par simple vraisemblance morphologique et topographique, on aura tendance, pour fixer celle-ci, à prendre appui sur le tracé courbe des rues modernes de l'Union et de la Cavalerie avec un éventuel prolongement par la rue de la Fraternité, en léger décalage à l'est, ou bien on pourra au débouché de la rue de la Cavalerie suivre un prolongement direct jusqu'à la rue du Cardinal de Fleury, en appui sur des limites en fond de parcelles parfaitement alignées.

- $\mathrm{Au}$ sud, on peut logiquement prolonger vers l'ouest l'alignement de la rue de l'Union par la rue Capiscolat (ou Capistolat selon les sources) puis la rue Joseph Galtier qui ferme le quartier épiscopal. Cette forme urbaine, en prolongement topographique logique du tracé retenu sur le flanc oriental, n'est pas datée. L'un de nous (Schneider 2003 : 221) a proposé de mettre en relation la structure ronde dite «du Capistolat » avec la topographie religieuse primitive du très haut Moyen Âge — possible premier groupe épiscopal avant un éventuel transfert à l'emplacement de la cathédrale actuelle, à la fin du VIII $^{\text {eme }}$ siècle ou au début du IX $^{\text {ème }}(?)$; il relève, par relation morphologique, « son antériorité sur toutes les autres unités de plan reconnues à l'intérieur de l'agglomération médiévale ». Cette ancienneté relative n'interdit cependant pas, en toute logique, de vieillir encore la structure pour en faire la limite de l'agglomération au Haut-Empire, au sud sud-est et à l'est. La structure du Capistolat apparaît d'ailleurs comme possiblement plus récente que la ligne continue formée par la suite rue du Capistolatrue de l'Union, qu'elle rompt et déborde légèrement au sud.

- Sur le flanc nord enfin, aucun élément précis ne milite en faveur de l'une des deux hypothèses que l'on peut retenir : soit une limite au droit de l'enceinte de 1351, soit une autre plus en retrait, en appui sur les rues du Cardinal de Fleury puis Vieille Commune. Toutes les découvertes archéologiques mentionnées plus haut, place Alsace-Lorraine et rue de la République en particulier, entrent dans le périmètre le plus étroit que nous aurons donc tendance à privilégier.

Ainsi définie, la surface potentiellement urbanisée pendant l'Antiquité couvre, suivant les variantes, une superficie maximale comprise entre 6 et 7 ha. Dans ce calcul n'est pas prise en compte la question de la densité du tissu ni celle d'éventuels vides de construction qu'il est impossible de mesurer.

La valeur n'a évidemment aucune commune mesure avec celle énoncée le plus couramment autour de 22 ha, que nous-même avons encore retenue et utilisée il y a peu (Garmy et al. sous presse). Elle devra désormais s'imposer, non seulement pour réfléchir sur la place de la ville dans sa Cité et son rôle dans 
l'organisation géopolitique de la province romaine, mais aussi pour fonder les futures stratégies d'interventions archéologiques sur la ville antique, sans préjudice bien sûr des nécessaires recherches de terrain sur les époques médiévales et modernes ni par ailleurs de celles, non moins indispensables, relatives aux nécropoles périphériques.

\section{Luteva en son territoire, parmi les Cités de Narbonnaise}

Cette superficie réduite situe la ville de Lodève parmi les plus petites capitales des Cités de la Narbonnaise pendant le Haut-Empire (fig. 13 et tab. infra). On sait, bien entendu, et l'on vient à nouveau d'en administrer une preuve patente, toute la difficulté qu'il y a à fixer la surface urbanisée des villes antiques. La documentation est souvent ancienne et pauvre, voire inexistante (c'est le cas notamment pour Carpentras et Cavaillon où aucune donnée fiable n'est disponible) et de toute façon se pose la question de savoir ce qu'on prend précisément en compte dans le calcul. Les villes dotées d'une enceinte, en apparence plus simples à cerner, constituent en fait des cas particulièrement épineux : certaines connaissant des occupations largement audelà des remparts (Vienne, Arles) quand d'autres intègrent un tissu urbain intra-muros très lâche, avec des vides d'occupation parfois considérables (Nîmes par exemple). Les chiffres que nous manipulons ici sont donc des approximations, issues des recherches les plus récentes quand elles existent, mais qui n'ont qu'une valeur relative aux seules fins de comparaisons à l'intérieur de l'armature urbaine de la province.

Les données utilisées sont les suivantes (surface exprimée en ha) :

\begin{tabular}{|c|c|c|}
\hline Aix & 65 & Guyon et al. $1998: 235$ \\
\hline Alba & 45 & $\begin{array}{l}\text { Gallia Informations } 1996: 22-32 \\
\text { Dupraz, Fraisse } 2001: 114-160\end{array}$ \\
\hline $\begin{array}{l}\text { Antibes ( } \text { " citadelle » + théâtre et amphithéâtre } \\
\text { extra-muros) }\end{array}$ & 1,4 & Rousselle $1976: 153$ \\
\hline Apt & 6 & Barruol $1968: 107$ \\
\hline Arles (rive gauche et Trinquetaille) & 75 & Heijmans, Sintès 1994 : 142-143 \\
\hline Avignon & 46 & Carru $1999: 114$ \\
\hline Béziers & 38 & Olive $1995: 212$ \\
\hline Carcassonne (cité intra-muros) & 7 & Passelac 1999 : 57-58 \\
\hline Carpentras & $?$ & Aucune donnée disponible \\
\hline Cavaillon & $?$ & Aucune donnée disponible \\
\hline Fréjus (intra-muros) & 46,5 & Rivet et al. $2000: 469$ \\
\hline Lodève & 2,5 & Supra 5 \\
\hline Marseille & 50 & Tréziny $2001: 53$ \\
\hline Narbonne & 100 & Coll. $2000: 11$ et 22 \\
\hline Nîmes (intra-muros) & 220 & Monteil $1999: 365$ \\
\hline Orange & 70 & Mignon et al. $1997: 175$ \\
\hline Riez & 15 & Borgard $1997: 361$ \\
\hline Ruscino (plateau) & 8,2 & Marichal $2002: 103$ \\
\hline Saint-Paul-Trois-Châteaux & 40 & $\begin{array}{l}\text { Gallia Informations } 1996: 75 \\
\text { Bel } 2002: 15\end{array}$ \\
\hline Toulouse (intra-muros) & 90 & De Filippo $2001: 212$ \\
\hline Vaison & 75 & Goudineau, de Kisch 1991: 21 \\
\hline Valence & 25 & $\begin{array}{l}\text { Réthoré } 1993 \text { : 105-111 } \\
\text { Gallia Informations } 1996: 81-83\end{array}$ \\
\hline Vienne (rive droite et rive gauche) & 300 & Le Bot Helly $2002: 105$ \\
\hline
\end{tabular}

Ainsi, Lodève prend place dans un groupe de villes capitales présentant une surface urbanisée inférieure à 10 ha, aux côtés d'Antibes (cas douteux), Apt, Carcassonne et Ruscino. Avec des valeurs de cet ordre, on est évidemment très loin des deux mastodontes de la province, Vienne et Nîmes (respectivement 300 et 220 ha), mais aussi de toutes les autres capitales que l'on peut ranger par commodité en deux classes : l'une allant de 15 à 50 ha où l'on trouve dans l'ordre Riez (15 ha), Valence (25 ha), Béziers (38 ha), Saint-Paul-Trois-Châteaux (40 ha), Alba (45 ha), Avignon (46 ha), Fréjus (46,5 ha) et Marseille (50 ha), l'autre de 65 à 100 ha, avec Aix (65 
ha), Orange (70 ha), Vaison et Arles (75 ha), Toulouse (90 ha) et Narbonne, capitale de la province (100 ha). Cette hiérarchie de surface des territoires urbanisés, extrêmement dilatée comme on le voit, ne coïncide apparemment ni avec le statut juridique de la ville, ni avec l'ancienneté de sa fondation, ni même avec ce que l'on peut connaître par ailleurs de l'échelle des critères d'urbanité. En revanche, il est suggestif de comparer la distribution des emprises au sol des villes capitales avec celle des territoires des Cités. On objectera, une nouvelle fois et à bon droit, que la plupart de ceux-ci sont fort mal définis, que la question a donné lieu et encore aujourd'hui, à des débats vifs et non tranchés qui sont d'ailleurs, au cas par cas, d'une grande utilité. Adoptant une solution de facilité, nous avons calculé géométriquement avec une tolérance d'erreur très grande, la surface du territoire de chaque cité de la province à partir du fond de carte évocateur que donna jadis Christian Goudineau (1980: 94) intitulé «Les civitates de Narbonnaise sous le Haut-Empire, telles qu'on peut les reconstituer en dépit de certaines incertitudes ». Ce fond, agrandi et redessiné a été tout simplement numérisé puis traité sous le logiciel Autocad (Georges Marchand fec.) qui a produit automatiquement la liste des surfaces du territoires. Certainement fausses dans le détail, les données, dont il ne faut pas retenir les valeurs absolues, sont cependant commodes dans la perspective d'une comparaison interne au corpus (fig. 13). Sans surprise on constate là encore des situations très contrastées, dans un rapport de 1 à près de 80 , entre le territoire le plus exigu, celui de Marseille et le plus vaste, celui de la Cité des Allobroges. Dans l'intervalle, toutes les valeurs existent.

Cependant, on doit souligner l'excellente corrélation (indice de corrélation $=0,80$ ) entre les deux distributions de superficies, ville/territoire. Malgré de très notables exceptions, dans un sens ou dans l'autre — Marseille et Toulouse illustrant chacune un cas extrême - il apparaît qu'il existe un rapport statistique direct entre la surface urbanisée des chefs-lieux de Cité et la superficie des territoires. Il ne faut sûrement pas surinterpréter cette observation. Les deux séries sont bien corrélées, ce qui signifie vraisemblablement qu'un niveau d'équipement urbain standard est requis en fonction de la taille du territoire à administrer mais force est de souligner à l'inverse que l'histoire particulière de la ville et de la formation du territoire peut engendrer de très forts écarts à la règle générale. On devra ainsi se pencher plus en détail sur les cas de capitales apparemment hypertrophiées par rapport à leur territoire : Orange, Saint-Paul, Avignon et bien sûr Nîmes et Marseille. A contrario, Toulouse, Vaison et Ruscino peuvent surprendre par la relative modestie du centre dans la Cité. Cet exercice, qui dépasserait les limites de notre propos, requerra la prise en compte de bien d'autres paramètres de hiérarchie urbaine que la simple surface urbanisée, même si on peut en retenir la pertinence au moins statistique au vu de ce qui précède.

Dans ce contexte, Lodève, avec une assiette urbaine de 6 à 7 ha et un territoire d'environ 750 à $800 \mathrm{~km}^{2}$ entre parfaitement dans la norme et correspond à la «maille» utilisée par le pouvoir romain dans l'agencement géographique du territoire provincial.

Pour autant, les fondements de la création de la Cité, aux confins de celles de Nîmes et de Béziers et sur les marges septentrionales de la Province demeurent largement inexpliqués. On a invoqué traditionnellement plusieurs facteurs susceptibles d'en rendre compte, au premier rang desquels le rôle de relais sur l'itinéraire reliant l'intérieur à la côte. C'est en particulier l'idée essentielle que retient P.-A. Février : "Une des fonctions de la ville a été d'être une étape sur la route qui reliait le littoral à la vallée du Tarn et à Rodez. La Table de Peutinger place Loteva entre Cessero et Condatomagus (La Graufesenque, Millau)» (Février 1989: 62). Nonobstant le fait que l'on puisse s'interroger, dans cette hypothèse, sur la nécessité de fonder une Cité pour en faire un relais routier, il faut aussi désormais prendre en compte les résultats de travaux récents sur le territoire de Lodève. Ils montrent notamment que la ville, contrairement aux idées reçues, n'est pas située sur un passage obligé pour joindre la plaine littorale et le causse (Guy, Delfieu 2003 : 61) mais, au contraire, que plusieurs itinéraires alternatifs et plus évidents au moins en termes d'orographie, à l'ouest par l'Escandorgue et à l'est par la côte d'Arboras, n'empruntent pas la vallée de la Lergue et évitent ainsi la ville (Garmy et al. sous presse). Ce qui revient à dire que l'itinéraire Saint-Thibéry-Millau par Lodève, attesté par la Table de Peutinger, peut manifester un tracé dérivé, une route « officielle » née peut-être avec la fondation de la colonie. Dans ce cas, la route répond à la nouvelle logique territoriale apparue avec la colonie et non l'inverse.

Tout aussi fragiles sont les conjectures sur l'existence d'un district minier autour de Lodève qui aurait pu stimuler l'organisation d'une nouvelle collectivité administrative. Une objection majeure réside dans le fait que les principales zones minières recensées, autour de Cabrières et de Lascours en particulier, sont situées hors de l'emprise du territoire de la Cité et leur période d'exploitation la plus active est largement antérieure à la fondation de la colonie (Clavel-Lévêque, Mauné 2002 : dossier V). Enfin, aucune des galeries repérées aux alentours immédiats de la ville n'est sérieusement datée de l'Antiquité, notamment pas celles découvertes encore tout récemment à la faveur du remodelage de l'autoroute A 75 au droit de l'agglomération et que l'on incline à croire au plus tôt médiévales.

Le site choisi pour implanter la ville fait également problème. Si l'on retient qu'aucun témoin antérieur au changement d'ère n'y a jamais été découvert, et ce ne sont pas quelques tessons erratiques de céramique campanienne A qui peuvent, en l'état, combler ce vide, il faut bien admettre que Lodève prend place sur un site neuf, au cours de la période augustéenne. Ces constatations archéologiques vont à l'encontre de l'hypothèse 
d'une organisation d'époque césarienne, à quoi renvoie le toponyme Forum Neronis, mais qui demeure sans consistance sur le terrain, à moins d'imaginer que le site primitif ait pu prendre place sur le Grézac, à quelques kilomètres à l'ouest de la ville. Mais le dossier sur ce gisement est par trop indigent pour pouvoir réellement retenir l'hypothèse.

Ce n'est pas le lieu pour ouvrir à nouveau le dossier concernant le statut des agglomérations de la liste de Pline l'Ancien et toutes les questions connexes, en particulier le maintien de communautés éventuellement autonomes dans le ressort des Cités de Nîmes et de Béziers (Christol 2002 : 86-87 vs Roth Congès 2003 : 549 564). Cependant, il n'est pas superflu d'examiner ici particulièrement le sort des communautés installées dans la basse vallée de l'Hérault (Mauné 2003 : 289-294). Si, conformément à ce qu'en pense Michel Christol (en dernier lieu 1998 : 209-211), Pline, dressant son inventaire au début de la période flavienne, utilise comme source principale un document dont on peut fixer la date de rédaction vers 27 av. J.-C., on a toutes raisons de considérer l'état géopolitique décrit comme celui qui était issu de la première organisation augustéenne, voire celui immédiatement postérieur à la fondation de la colonie romaine de Béziers en 36 av. J.-C. Du sud vers le nord, on a donc pour cette période, suivant l'axe de la vallée de l'Hérault: Agde (Agatha quondam Massiliensum), Cessero, Piscince, Lutevani et immédiatement à l'ouest (Christol 1998: 213) le territoire des Ruteni (provinciales). Peu importe ici l'état des connaissances sur chacune de ces agglomérations : Agde dont la consistance pour la période romaine est problématique (Ugolini 2002 : 364), Cessero que l'archéologie peine à caractériser (Mauné 2002: 340) et Piscince dont même la localisation fait débat (Barruol $2002: 29$ et 31 vs Mauné 2003 : 288). Toujours est-il que sur l'ensemble de ces communautés mentionnées par Pline, un temps autonomes et jouissant du droit latin, à la frontière des territoires des colonies de Nîmes et de Béziers, seule Lodève ne fut pas déclassée comme les autres mais connut au contraire un destin singulier, faisant mieux que tirer son épingle du jeu pour accéder au statut de colonie latine, sans doute sous Claude comme en témoigne sa titulature. Sort hors norme dans le contexte local, d'autant plus que la réalité archéologique de l'agglomération des Lutevani antérieurement au changement d'ère est réellement intangible. Tout se passe comme si l'organisation d'une Cité des Lutevani, avec sa capitale créée ex nihilo sous Auguste, répondait essentiellement à une décision géopolitique romaine, dont le fondement nous échappe mais qui n'en est pas moins attestée par les faits. Consacrant éventuellement une communauté (arécomique ?) antérieure, l'oppidum latinum des Lutevani n'existe peut-être dans la formula d'origine que sous forme d'intention, dont rendrait d'ailleurs mieux compte la seconde appellation de Forum Neronis. Ressortissant d'une pure volonté politique du pouvoir colonial qui y trouve assurément un intérêt continu et à long terme, Lodève, au moment où ses voisines, un temps distinguées, sont absorbées dans l'anonymat du territoire des grandes Cités voisines, est non seulement préservée mais, peu après, élevée au rang supérieur de colonie.

\section{Insertion de la capitale dans son réseau de peuplement}

Si l'espace urbain de Lodève durant l'Antiquité demeure, comme on l'a vu, très imparfaitement documenté, la connaissance de l'espace rural de la Cité a fait des progrès considérables depuis la courte synthèse consacrée à Luteva par A. L. F. Rivet (1988 : 160-161). En effet, on a repéré aujourd'hui plus d'une cinquantaine d'établissements ruraux de la période républicaine et du Haut-Empire, très majoritairement implantés dans la partie sud-est du territoire, dans le bassin moyen de l'Hérault, en rive droite, là où la Lergue se jette dans le fleuve (Schneider, Garcia 1998).

\subsection{Avant la colonie}

Aux confins des territoires de trois groupes indigènes (Rutènes, Volques Arécomiques et Volques Tectosages) cet espace de plaine, circonscrit par la haute muraille de la Séranne au nord, la terminaison orientale de la Montagne noire et les coulées basaltiques de l'Escandorgue à l'ouest, jouit d'un réel dynamisme bien avant la fondation de Luteva. La spécificité de cette zone est d'autant plus saisissante qu'elle s'inscrit dans un net contraste avec la situation de la rive opposée de l'Hérault. Alors qu'ici, la plupart des oppida indigènes sont abandonnés avant les $\mathrm{II}^{\mathrm{ème}}$ et $\mathrm{I}^{\mathrm{er}} \mathrm{s}$. av. notre ère, ceux de la rive droite, en pays lodévois donc, connaissent à cette époque de nouveaux développements (fig. 14). À Nébian, dans la vallée de la Dourbie, le Roc du Cayla porte alors, sur près de 6 ha l'une des occupations les plus denses de son histoire. Moins d'une dizaine de kilomètres plus au nord, l'oppidum de Cornils à Lacoste, établi au débouché de la Lergue dans le bassin de l'Hérault connaît le même processus, renforcé de surcroît par l'émergence d'un quartier bas, vraisemblablement associé à un itinéraire permettant de rejoindre le pays rutène. À côté de ces pôles traditionnels de peuplement apparaissent, à la même époque, de nouveaux habitats groupés qui sont également inconnus en rive gauche du fleuve. Un premier groupe d'établissements correspond à une nouvelle génération d'oppida et désigne donc des habitats perchés. Cette situation paraît presque anachronique, mais la forme de ce perchement répond à un mode d'implantation qui semble différent des pratiques du $\mathrm{I}^{\text {er }}$ âge du fer. Il ne s'agit plus, en effet d'investir des 
collines ou des éperons d'altitude moyenne, souvent associés à des vallées et à la juxtaposition de terroirs complémentaires. Bien au contraire, les nouveaux sites occupés sont de véritables bastions géologiques qui figurent parmi les reliefs majeurs et s'imposent dans le paysage ceinturant la rive droite de l'Hérault. Ainsi, le nouvel établissement du Rocher des Vierges à Saint-Saturnin culmine à plus de $500 \mathrm{~m}$ d'altitude et constitue une sorte de verrou entre plaine fluviale et piémont du Massif central. Celui du mont Liausson entre Mourèze et Liausson atteint lui aussi plus de $500 \mathrm{~m}$ d'altitude et domine à la fois la dépression centrale du Lodévois au niveau de la zone du Salagou au nord et le paysage si particulier du cirque de Mourèze au sud. Malgré leur position en retrait par rapport au bassin de l'Hérault et aux meilleurs sols, ces nouveaux habitats groupés n'en sont pas moins ouverts sur des activités d'échange comme en témoigne l'abondance des fragments d'amphores italiques recueillis sur leur emprise. Enfin un phénomène plus original se produit parallèlement avec, parmi les nouveaux pôles qui émergent au cours des II $^{\mathrm{eme}}$ et $\mathrm{I}^{\mathrm{er}}$ s. av. J.-C., des agglomérations de plaine que l'enquête archéologique commence tout juste à révéler. Au nord du secteur, toujours en rive gauche de la Lergue, à moins de $4 \mathrm{~km}$ de l'établissement perché du Rocher des Vierges, une nouvelle agglomération s'installe dans la plaine de Saint-Saturnin. Inconnue il y a peu de temps encore, ce site des Aulas a été mis en évidence par les prospections conduites dans le cadre du PCR lodévois (Rascalou 1997 ; Rascalou, Schneider 2002).

À une époque donc où Lodève n'existe pas encore, puis de manière contemporaine à l'entrée en lice de Forum Neronis, la partie orientale du territoire de la Cité, riveraine de l'Hérault, est déjà équipée d'une armature urbaine dense et diversifiée.

\subsection{La situation du Haut-Empire à la fin de l’Antiquité (fig. 15)}

L'établissement des Aulas qui s'étend sur une superficie comprise entre 6 et 9 ha a été occupé, contrairement à la dernière génération d'oppida, au delà de l'époque augustéenne et a connu son principal essor sous le Haut Empire. Durant la première moitié du I ${ }^{\mathrm{er}}$ s. ap. J.-C. notamment, deux quartiers spécialisés établis dans la périphérie de l'agglomération sont associés à des ateliers de fabrication de céramique sigillée. L'existence, à proximité des Aulas, d'un axe de communication permettant depuis le Montpelliérais de rejoindre le causse du Larzac et le pays rutène par la côte dite d'Arboras sans emprunter le couloir de la Lergue (cf. supra) explique sans doute une partie de l'essor de l'agglomération. Cet itinéraire, insoupçonné il y a peu encore, marginalise Lodève et constitue peut-être l'un des principaux axes sur lequel s'est appuyée la diffusion des sigillées rutènes vers la zone méditerranéenne. Il s'agit là du moins d'une piste de réflexion qu'il conviendrait d'approfondir, car durant la première moitié du $\mathrm{I}^{\mathrm{er}}$ s., l'agglomération des Aulas et ses deux officines ont pu constituer une succursale de la Graufesenque chez les Lutevani comme il en était probablement de l'atelier des Roziers chez les Gabales (Ferdières 2003 : 219). Au sud du bassin de l'Hérault, dans le secteur de Clermontl'Hérault, cette fois-ci, en rive droite de la Lergue, on observe le même essor. Non loin de l'oppidum de Cornils dont les limites anciennes sont outrepassées par un quartier bas, émerge là aussi une nouvelle agglomération de plaine, dès l'époque républicaine et le développement de celle-ci, comme aux Aulas, se prolonge durant le HautEmpire. Sur une dizaine d'hectares, l'établissement de Peyre Plantade a bénéficié de très récentes fouilles (Pomarèdes dir.) révélant une phase d'épanouissement de l'ensemble dans les dernières décennies du $\mathrm{I}^{\mathrm{er}} \mathrm{s}$. av. et durant le $\mathrm{I}^{\mathrm{er}}$ s. ap. J.-C. Quartier artisanal, unités de production agricole, surtout viticoles, faubourgs, fonctions de service (relais routier), mais aussi habitations à cour intérieure, évoquent l'image d'une place jouant un rôle central comme lieu de concentration et de redistribution des productions locales et des importations, d'autant qu'à partir du milieu du $\mathrm{I}^{\mathrm{er}}$ s. ap. J.-C., un véritable chapelet d'établissements dispersés est implanté dans un rayon d'un à deux kilomètres autour de l'agglomération (Bermond et al. 2002 : 251-256).

Dans cette zone de piémont, l'essor de deux oppida du premier âge du fer au cours des $\mathrm{II}^{\text {ème }}$ et $\mathrm{I}^{\mathrm{er}} \mathrm{s}$. av. n. ère, de même que l'émergence d'une nouvelle génération d'habitats groupés de hauteur et l'apparition de deux « agglomérations » de plaine font de la rive droite du bassin moyen de l'Hérault, une zone particulièrement dynamique dont l'identité se renforce du contraste qu'offre la rive gauche du fleuve qui n'a pas connu un tel sort. C'est sans doute un trait qu'il convient de garder à l'esprit parce qu'il fonde la spécificité et la personnalité d'un territoire somme toute restreint situé dans un secteur de confins entre Nîmes et Narbonne, Méditerranée et Massif central. Dans ce contexte, Lodève où l'on n'a relevé jusqu'à présent aucune strate préaugustéenne apparaît comme une fondation «tardive », qui plus est, située en net retrait de cette zone dynamique. Nouvelle capitale politique, celle-ci n'assure pas la promotion d'un établissement antérieur, pas plus qu'elle ne semble avoir bridé le développement des places locales de la plaine. Lodève apparaît d'abord comme une décision administrative qui établit une nouvelle hiérarchie coiffant les réseaux locaux de peuplement dont les pôles paraissent, en première analyse, avoir surtout tenu des fonctions économiques, artisanales et marchandes. La position de la capitale, enfoncée au plus près de la barrière rocheuse du causse du Larzac, inscrit dès lors l'identité de ce territoire dans un espace géographique de transition. Entre causse et vallée fluviale, Lodève est d'abord la capitale politique du pays de Lergue, plus qu'elle n'est un pôle démographique ou d'échange. Cet affluent de l'Hérault constitue en définitive la véritable épine dorsale autour de laquelle s'est construit le territoire civique des Lutevani et la question de la centralité de la capitale trouve plus volontiers un écho dans la 
position médiane qu'elle occupe sur son parcours que dans son insertion, bien imparfaite dans les réseaux de communication et des pôles de peuplement local. Un pied en plaine, un pied en montagne, Lodève, bien modeste capitale du pays de la Lergue réalise finalement l'unité politique d'un espace géographique improbable du fait de ses contrastes accentués.

Le geste politique romain pour pragmatique qu'il soit, relève finalement dans le temps long d'une belle fortune. L'accueil d'un siège épiscopal au moins depuis le premier quart du $\mathrm{V}^{\text {ème }}$ s. suggère en effet que le poids politique de la capitale a été consolidé au cours des derniers siècles de l'Antiquité. En l'état actuel de la documentation, ce renforcement de la ville semble devoir être associé à l'échec du processus d'urbanisation de la zone sud-est du territoire. Alors que les oppida du Cayla, du mont Liausson et du Rocher des Vierges sont abandonnés avant l'époque augustéenne, les deux principales agglomérations de la plaine, Peyre Plantade et les Aulas montrent de leur côté des signes de faiblesse dès la fin du $\mathrm{II}^{\text {ème }} \mathrm{s}$. de notre ère et ceux-ci ne font que s'accentuer au cours des III ${ }^{\text {ème }}$ et IV $^{\text {ème }}$ s. Au V ${ }^{\text {ème }}$ s., seul le site de Cornils, véritable verrou au débouché de la Lergue dans le bassin de l'Hérault, peut encore désigner un habitat groupé. L'implantation de ce site marque d'ailleurs une sorte de repli sur le couloir de la Lergue, un possible refermement autour de la cité. Celle-ci a telle bénéficié d'un apport démographique au cours des II $^{\text {ème }}-V^{\text {ème }} \mathrm{s}$. ? La documentation archéologique est bien mince pour l'affirmer. L'échelle restreinte du territoire des Lutevani se révèle néanmoins comme l'un des principaux atouts de la cité à la fin de l'Antiquité. Aux confins des «centres » rutène, nîmois et, dans une moindre mesure, biterrois, la maille est particulièrement pratique et peut fournir le cadre médian dans lequel se resserrent durant tout le premier Moyen Âge les écarts hiérarchiques, d'autant qu'aucun autre pôle urbain ne s'était finalement constitué dans le territoire des Lutevani.

\subsection{Le destin de la capitale au cours du premier Moyen Âge}

On sait peu de choses sur le processus de christianisation de la cité. La dédicace de la cathédrale à Saint Geniès, dont le martyre en Arles est connu notamment par un récit de Grégoire de Tours, suggère une influence de la cité primatiale. Cela est d'autant plus probable que l'évêché de Lodève entre précisément dans l'histoire au sujet d'une affaire qui avait opposé en 422, l'ordo des clercs de la cité à Patroclus évêque d'Arles parce que celui-ci, fort de son récent titre de primat avait installé un prélat de son choix sur le siège lodévois. C'est peut-être dans ce contexte particulier des premiers conflits de pouvoir et d'influence entre Arles, Vienne et Narbonne qui traduisent surtout l'incertitude des cadres administratifs que Lodève acquiert la dimension épiscopale. Dans cette affaire, la dédicace de la cathédrale au martyr arlésien apparaît d'abord comme l'écho d'une influence de cette cité en Narbonnaise mais l'on ne peut préciser si celle-ci fut la conséquence directe des prétentions de Patroclus ou liée à l'action de ses prédécesseurs.

Toujours est-il que la diffusion du culte de Saint Geniès trouve une réelle consistance en Lodévois et sur ses marges. La fouille de sauvetage engagée par l'équipe du PCR autour de l'église Saint-Geniès-de-Litenis vient de montrer que le sanctuaire roman du XII ${ }^{\mathrm{eme}} \mathrm{s}$. avait été précédé par un sanctuaire plus ancien que l'on rattache aux $\mathrm{V}-\mathrm{VI}^{\text {èmes }} \mathrm{s}$. Voilà l'un des plus anciens témoignages de la christianisation des campagnes lodévoises. Il n'est sans doute pas anodin de noter que ce sanctuaire fut dédié à saint Geniès comme la cathédrale et qu'il demeure encore associé à un noyau fiscal au début du $\mathrm{IX}^{\mathrm{ème}}$ s., signes possibles que ces terres relevaient de l'Église lodévoise à la fin de l'Antiquité (Schneider 2000 : 61). La carte de la diffusion des vocables des saints arlésiens que vient de dresser M. Baudat (Guyon, Heijmans 2002 : 155) peut d'ailleurs être complétée par d'autres exemples. En Lodévois, dans le bassin du Salagou, l'église de la villa de Salasc, associée au patrimoine de la cathédrale dès 884 est, elle aussi, placée sous le vocable de Saint Geniès (C. Lodève, V : 3). On peut également évoquer cette église Saint-Geniès-des-Fours sur le causse du Larzac ou ce toponyme Saint-Geniès à proximité du castrum de Caux en Biterrois, biens qui ressortissaient au moins depuis le XII ${ }^{\mathrm{eme}}$ s. du temporel de l'Église lodévoise. On note encore dans la moyenne vallée de l'Hérault, en rive gauche l'existence à Gignac d'un site de l'Antiquité tardive associé à un quartier cadastral dénommé Saint-Geniès qui indique peut-être l'existence d'un sanctuaire paléochrétien inconnu de la documentation écrite médiévale et moderne.

La piste est bien mince pour évoquer cette Église lodévoise des premiers temps chrétiens, mais il nous semble qu'elle contient le souvenir de l'influence de la cité arlésienne dans cette partie de la Narbonnaise durant les dernières décennies du $\mathrm{IV}^{\text {ème }} \mathrm{s}$. et au tout début du $\mathrm{V}^{\text {ème }} \mathrm{s}$.

La documentation du $\mathrm{VI}^{\text {ème }}$ s. ouvre quant à elle une autre perspective, celle qui lie plus clairement le destin de ce territoire à celui du pays rutène, ou du moins à un horizon continental et septentrional mieux avéré. En 535 , l'évêque Deutaire siège au concile de Clermont en Auvergne ce qui laisse entendre que Lodève avait été détaché de la nouvelle Septimanie wisigothique pour être intégré au royaume des Francs. La conquête de ce territoire semble liée, deux à trois années plus tôt, à une incursion des troupes de Théodebert qui enlèvent deux castella wisigoths, Dio et Cabrières sur les marges du Lodévois, dans la montagne biterroise. L'affaire concerne directement l'histoire lodévoise parce que l'élection de Deutaire pourrait être liée à cet épisode. C'est en effet une certaine Deoteria, peut-être une parente de l'évêque, qui livra la place de Cabrières à Théodebert. En tout cas, il est significatif de constater que parmi le maigre patrimoine de l'Église lodévoise du IX ${ }^{\text {ème }}$ s. se trouvent 
deux villare (Bardens et Nizas) situés en Biterrois non loin du castrum de Cabrières. L'affaire nous montre aussi le rôle nouveau joué par ces forteresses tardo-antiques dans la défense du territoire et doit attirer l'attention sur deux réoccupations de hauteur de l'espace lodévois. Dans le couloir de la Lergue, Cornils joue un rôle de verrou entre plaine et premiers contreforts du causse tandis qu'au nord de Lodève, la réoccupation du Grézac assure un contrôle possible des passages de l'Escandorgue principal accès au Larzac et au pays rutène depuis la cité.

Jusqu'à l'épiscopat de saint Fulcran dans les dernières décennies du $X^{\text {ème }}$ s. l'histoire de la ville et de son territoire demeure ensuite dans l'ombre la plus épaisse. Avant la fin du VI ${ }^{\text {ème }}$ s., la capitale lodévoise est intégrée au royaume de Tolède, mais la liste épiscopale s'interrompt entre 683 et 817 . Même avec la restauration carolingienne, le patrimoine de l'Église épiscopale paraît bien médiocre. Sur les marges occidentales du territoire l'émergence du nouveau monastère d'Aniane dans le dernier tiers du VIII ${ }^{\text {ème }}$ s., puis de sa cella de Gellone (StGuilhem-le-Désert) marque des transferts de terres considérables. Complexes fiscaux (Litenis à Saint-Jean-deFos et Coussenas à Saint-André), églises et principaux domaines ruraux de la plaine lodévoise (Ceyras, SaintFélix-de-Lodez, Canet, Marecomitis...) passent alors aux mains des moines, ce qui ne peut que souligner la faiblesse de la cité épiscopale et porte interrogation sur sa situation matérielle. Une capitale réduite à une cathédrale ? Cela paraît d'autant plus probable que Lodève, contrairement à Nîmes, Béziers, et même à de nouvelles cités comme Maguelone et Agde, paraît tout au long des VIII-IX ${ }^{\text {èmes }}$ s. dépourvue d'un siège comtal propre. L'émergence de deux pôles monastiques redistribue les réseaux locaux de peuplement au détriment de la cité et crée de nouvelles centralités qui semblent à nouveau marginaliser Lodève. Un mouvement monastique se produit bien autour du noyau épiscopal, mais ces modestes cellce des vallées de Lauroux (Saint-Pierre), de Pégairolles (Saint-Jean) et peut-être même des abords de la cité (Saint-Amans et Saint-Martin) n'ont pas la richesse et le rayonnement de Saint-Sauveur d'Aniane et de Saint-Sauveur de Gellone. L'action des prédécesseurs de saint Fulcran dans la vie de la cité demeure difficile à cerner. Durant la première moitié du IX $^{\text {ème }}$ s. Sisemond et Tatila sont à l'origine d'une modeste restauration du temporel de l'Église qui nous montre d'abord que la cathédrale est à la tête de quatre ou cinq cellae établies dans les vallées sous-peuplées autour de Lodève auxquelles ne s'ajoutent à la fin du siècle que quatre villae et deux villare. Autour des années 870-877, la vita de saint Georges nous apprend l'origine rouergate, de cet autre évêque lodévois qui fut auparavant moine à Conques et à Vabres. Son nom suggère d'ailleurs une proximité avec des agents des comtes de Rouergue, autre témoignage possible du lien qui unit l'aristocratie du Lodévois à celle du pays rutène (Belmon 1991 : 85-86).

Ce n'est cependant qu'à partir du dernier tiers du $X^{\text {ème }}$ s., sous l'épiscopat de saint Fulcran que la « ville » atteint un nouveau seuil de développement, marqué principalement par une restructuration du cœur de la cité. La vieille cathédrale de Saint-Geniès fut agrandie, dotée d'un riche patrimoine et consacrée en 975. Fulcran établit également un monastère à Lodève, autour d'une ancienne église Sainte-Croix (souvenir d'un groupe épiscopal primitif ?) que son prédécesseur Théodoric aurait dédiée au Sauveur dans la première moitié du $\mathrm{X}^{\text {ème }} \mathrm{s}$. Si l'on en croit Bernard Gui, l'institution de ce nouvel établissement fut le résultat de la translation d'un monastère situé en dehors des murs de la cité, peut-être cette cella de Saint-Amans ou de Saint-Martin mentionnée dans les diplômes du début du IX ${ }^{\text {ème }}$ s. Quoi qu'il en soit, l'agrandissement de la cathédrale et l'établissement d'un monastère à ses côtés sont le signe d'une revitalisation du cœur urbain qui fonde la nouvelle organisation de la ville médiévale. C'est en tout cas à partir de ce noyau marquant désormais la limite occidentale de l'agglomération que celle-ci s'étend progressivement jusqu'à la confluence de la Lergue et de la Soulondres. Ce réveil urbain du $X^{\text {ème }}$ s. est contemporain de la mise en place d'un dispositif de protection militaire des noyaux d'habitat et des patrimoines les plus proches de la cité et saint Fulcran lui-même paraît avoir joué un rôle dans l'établissement de ce dispositif. À quelques kilomètres à peine de Lodève, dans les vallées du piémont de Larzac des tours sont édifiées, avant 988, à Aubaigues, Soubès et Fozières, et ces structures créent une nouvelle forme de polarité autour de la cité. Mais Lodève elle-même ne semble pas avoir possédé de château avant le XII ${ }^{\text {ème }}$ s. et le pagus est également dépourvu de vieilles forteresses carolingiennes comme il en existait dans l'arrière-pays montagnard du Biterrois ou de la région de Nîmes. C'est peut-être le signe de l'établissement tardif d'un pouvoir civil dans ce petit territoire, pagus mineur qui ne semble pas avoir possédé de siège comtal ou vicomtal propre avant le milieu du $X^{\text {ème }}$ s. Le plus puissant et le plus ancien château du Lodévois n'émerge d'ailleurs pas avant cette date. Établi sur l'éperon calcaire des Deux-Vierges à Saint-Saturnin, que l'on a vu occupé une première fois aux $\mathrm{II}^{\text {ème }}-\mathrm{I}^{\mathrm{er}} \mathrm{s}$. av. notre ère, ce castrum occupe alors une position de charnière entre plaine, causse et bassin intérieur de la Lergue et marque la première phase de la territorialisation d'un pouvoir aristocratique local.

On retiendra surtout que ce processus paraît ici indépendant du fait urbain mais participe d'une nouvelle forme d'encadrement du territoire rural. Dans la longue tradition du haut Moyen Âge, Lodève demeure jusqu'au XII ${ }^{\text {ème }}$ s. un pôle épiscopal, le cœur identitaire d'un petit pays plutôt qu'une véritable capitale urbaine et démographique. Cet état est-il d'ailleurs radicalement différent de ce qu'il fut au cours de l'Antiquité ? 